\title{
Ultrasound-Guided Local Anesthetic Infiltration Between the Popliteal Artery and the Capsule of the Posterior Knee (IPACK) Block for Primary Total Knee Arthroplasty: A Systematic Review of Randomized Controlled Trials
}

\section{Ryan S D'Souza \\ Brendan J Langford \\ David A Olsen \\ Rebecca L Johnson}

Department of Anesthesiology and Perioperative Medicine, Mayo Clinic Hospital, Rochester, MN, USA
Correspondence: Rebecca L Johnson Department of Anesthesiology and Perioperative Medicine, Mayo Clinic College of Medicine, 200 First St. SW, Rochester, MN, USA, 55905

Email johnson.rebecca@mayo.edu

\begin{abstract}
Posterior knee pain after total knee arthroplasty (TKA) is common despite multimodal analgesia and regional anesthesia use. This review included randomized controlled trials (RCTs) comparing analgesic outcomes after inclusion of local anesthetic infiltration between the popliteal artery and capsule of the knee (iPACK) block versus pathways without iPACK. Electronic databases (MEDLINE, Cochrane Library, Web of Science, Scopus) were searched from inception to 10/11/2020. Eligible studies evaluated iPACK use on primary outcomes: opioid consumption and pain scores with movement. Secondary outcomes included rest pain, patient satisfaction, length of stay (LOS), gait distance, knee range of motion (ROM), and complications. Bias and quality were appraised using the Cochrane Risk of Bias tool and Grading of Recommendations, Assessment, Development, and Evaluations (GRADE) guidelines. Eight RCTs (777 patients) were included. iPACK block use demonstrated similar opioid consumption in the PACU (4/7 RCTs) and 24 hours after TKA (5/7 RCTs) compared to without iPACK (moderate-quality GRADE evidence). Additionally, iPACK block use demonstrated lower movement pain scores in PACU (3/5 RCTs) but similar or higher pain scores after 24 hours (5/7 RCTs; lowquality GRADE evidence). Studies consistently reported no difference in gait distance (4/4 RCTs) or complications (7/7 RCTs) between treatment arms (high-quality GRADE evidence), although differing effect estimates were observed with resting pain, satisfaction, LOS, and knee ROM. This review provides a foundation of knowledge on iPACK efficacy. While evidence does not currently support widespread inclusion of iPACK within enhanced recovery pathways for TKA, limitations suggest further study is warranted.
\end{abstract}

Keywords: regional anesthesia, analgesia, total knee arthroplasty, pain, treatment outcome, systematic review, meta-analysis

\section{Introduction}

Total knee arthroplasty (TKA) is the most common surgical procedure in the United States and is predicted to reach 3.48 million surgeries annually by $2030 .{ }^{1}$ There is concern that extreme knee pain immediately post-surgery has been reported in approximately half of TKA patients. ${ }^{2-4}$ Optimal postoperative knee analgesia is not only important for patient comfort and satisfaction but also essential for 
accelerating mobilization, functional recovery, and hospital discharge. ${ }^{5}$ There is an increasing emphasis on multimodal analgesia and motor-sparing regional anesthetic blocks to facilitate earlier ambulation and provide superior pain control as we strive for shorter hospital stays and same-day discharges for patients. ${ }^{6}$

Randomized controlled trials (RCTs) and metaanalyses have considered a combination of femoral and sciatic nerve blockade for analgesia for TKA, although these more proximal blocks are associated with prolonged motor weakness and delayed ambulation. ${ }^{7}$ Surgeonadministered periarticular injections (PAI) may be motorsparing, but may also provide incomplete analgesia. ${ }^{8}$ The adductor canal block (ACB) can spare quadriceps muscle strength; however, due to its predominantly anteromedial coverage of the peri-patellar and intra-articular aspects of the knee, ACB fails to alleviate posterior knee pain which may be severe in intensity. ${ }^{9-11}$

Ultrasound-guided infiltration of local anesthetic in the interspace between the popliteal artery and posterior capsule of the knee (iPACK) is a novel regional anesthetic modality for posterior knee analgesia. The iPACK block targets the medial and lateral genicular nerves, and other articular branches innervating the posterior aspect of the knee joint which in theory has less motor and sensory blockade below the knee than the more proximal sciatic nerve block. ${ }^{12-14}$ Despite an increasing number of recent clinical trials on the iPACK block, there have been few efforts to systematically synthesize the efficacy of the iPACK block.

The aim of this systematic review was to examine and summarize all available RCTs on the use of the iPACK block in patients undergoing TKA, focusing on comparisons of analgesic outcomes and functional recovery with other regional anesthetic modalities. We hypothesized that compared to those not receiving an iPACK block as an adjunct, patients receiving an iPACK block would report lower pain scores, consume fewer opioids, achieve improved ambulation, and report superior satisfaction with analgesia in the postoperative period with a similar incidence of adverse events.

\section{Materials and Methods}

\section{Search Strategy}

The study protocol was registered under the PROSPERO International prospective registry of systematic reviews. ${ }^{15}$ We adhered to the Preferred Reporting Items for Systematic Reviews and Meta-Analysis (PRISMA) guidelines. ${ }^{16}$ We searched articles identified from various electronic databases, including PubMed (1966-October 2020), Cochrane Central Register of Controlled Trials databases (1993-October 2020), Web of Science (1980October 2020), Scopus (1996-October 2020), and also hand-searched reference lists of identified publications. Broad MeSH terms and Boolean operators were selected for each database search, including terms and synonyms for iPACK, nerve block, total knee arthroplasty, analgesia, pain, opioid consumption, patient satisfaction, and length of stay. This search strategy was verified by a librarian experienced in systematic review methods and is displayed in the Supplemental File.

\section{Study Selection}

Inclusion criteria encompassed: RCTs that compared patients undergoing TKA who received an iPACK block and control patients who received another peripheral nerve block or PAI (without PACK); and studies that measured a primary or secondary outcome of pain score or postoperative opioid consumption. While the PAI block involves surgeon-delivered infiltration into the posterior capsule of the knee, it also involves infiltration into the lateral femoral periosteum, medial femoral periosteum, and within the knee capsule and skin. ${ }^{17}$ The diffuse infiltration technique of the PAI is considered as a distinct intra-articular block compared to the more directed and ultrasound-guided iPACK peripheral nerve block.

Exclusion criteria comprised of: non-peer reviewed publications, certain study designs (ie observational studies, non-randomized controlled trials, case reports, case series, review articles, letters to the editor), and nonhuman trials. Two authors (RSD and BL) independently selected abstracts as well as full-text articles from the above listed databases using the aforementioned search strategies, and a third author (RLJ) adjudicated discrepancies.

\section{Data Extraction}

The following data were extracted: (1) demographic data of participants; (2) type of hospital setting (eg academic, community hospital); (3) local anesthetic dosage in iPACK and other utilized lower extremity peripheral nerve blocks; (4) location of iPACK block (proximal or distal) and (5) clinical outcomes of interest. The dual primary outcomes of interest were total opioid consumption in oral morphine equivalents (OMEs) in the postanesthesia care unit (PACU) and up to 24 hours 
postoperatively, and patient-reported pain score with movement up to 24 hours postoperatively. Secondary clinical outcomes of interest included patient-reported pain score at rest, patient satisfaction with analgesia, hospital length of stay, gait distance, knee range of motion (ROM), and complications (allergic reactions, nerve injury, nausea/vomiting, and respiratory depression). For each included study, two reviewers (RSD and BL) extracted all relevant data independently, and any disagreement was resolved by a third reviewer (RLJ).

\section{Assessment of Risk of Bias}

The quality of studies was independently evaluated by two reviewers (RSD and BL) utilizing guidelines from the Cochrane Collaboration ${ }^{18}$ for randomized controlled trials. Disagreements were adjudicated by a third author (RLJ). Biases were assessed in the following domains for RCTs only: random sequence generation, allocation concealment, blinding of participants and personnel, blinding of outcome assessment, attrition bias due to missing data, reporting bias, and other biases (eg departures from intended interventions, cross-contamination, confounding factors). Each domain was assigned a grade of low risk, high risk, or unclear risk.

\section{Assessment of Quality of Evidence}

Utilizing the GRADEpro software (http://gradepro.org) and following the Grading of Recommendations, Assessment, Development, and Evaluation (GRADE) quality assessment criteria, ${ }^{19}$ two reviewers (RSD and BL) independently assessed the level of evidence for each outcome across all included studies (high, moderate, low, or very low). RCTs are categorized as high-level evidence, although level of evidence can be downgraded because of deficiencies in domains of risk of bias, ${ }^{20}$ inconsistency, ${ }^{21}$ indirectness,${ }^{22}$ imprecision, ${ }^{23}$ and publication bias. ${ }^{24}$

\section{Data Analysis}

Given the substantial clinical heterogeneity of included RCTs, we deemed a meta-analysis to be inappropriate. Instead, we present a qualitative synthesis of the findings from included RCTs for primary and secondary outcomes of interest. The dependence assumption was not violated as observations within each study were independent of each other.

\section{Results}

\section{Search Results}

The search strategy identified 2180 unique citations. After independent and duplicate screening by two authors (RSD and $\mathrm{BL}$ ), eight RCTs ${ }^{13,25-31}$ were identified that fulfilled eligibility criteria. Collectively, these eight RCTs consisted of 777 patients (377 patients received the iPACK block; 400 patients in the control cohort received another modality of peripheral nerve blockade or PAI for knee analgesia). Figure 1 displays the PRISMA flow diagram of the study selection and inclusion process.

\section{Risk of Bias and Quality Assessment}

A summary of findings table with GRADE quality of evidence for each outcome and reason for quality assignment is presented in Table 1. Bias assessment for all included studies is summarized in Figure 2. All eight RCTs ${ }^{13,25-31}$ generally demonstrated a low risk of bias in random sequence generation and allocation concealment, blinding of outcome assessment, incomplete outcome data, and selective reporting. In terms of blinding of participants, some RCTs ${ }^{13,25,26}$ displayed a high risk for bias because although they blinded all surgeons, recovery room and floor nurses, research assistants, statisticians, and patients, it was impossible to blind the anesthesiologist performing the ultrasound-guided iPACK block who may have participated in the study. Furthermore in one study ${ }^{13}$ operating room nurses provided the local anesthetic medication to the anesthesiologist based on the type of regional block and thus were also not blinded. Other sources of bias included no true control group without a posterior knee block, and unequal dose of local anesthetic between study arms.

\section{Study Group Characteristics}

Table 2 presents the description of study characteristics. Exposure variables were heterogeneous across studies. Three RCTs ${ }^{26-28}$ compared iPACK block plus ACB versus ACB alone (with or without sham iPACK), two RCTs ${ }^{30,31}$ compared iPACK block plus ACB plus PAI versus ACB plus PAI plus sham iPACK, while other studies each compared unique combinations of peripheral nerve block or PAI block. For the primary anesthetic, five studies ${ }^{13,25,28,29,31}$ administered neuraxial anesthesia, two studies ${ }^{26,27}$ either administered general anesthesia or neuraxial anesthesia per the discretion of the primary anesthesia team, and one study administered general anesthesia only. ${ }^{30}$ All RCTs excluded patients who had a history of chronic pain or utilized preoperative opioids. $^{13,25-31}$ 


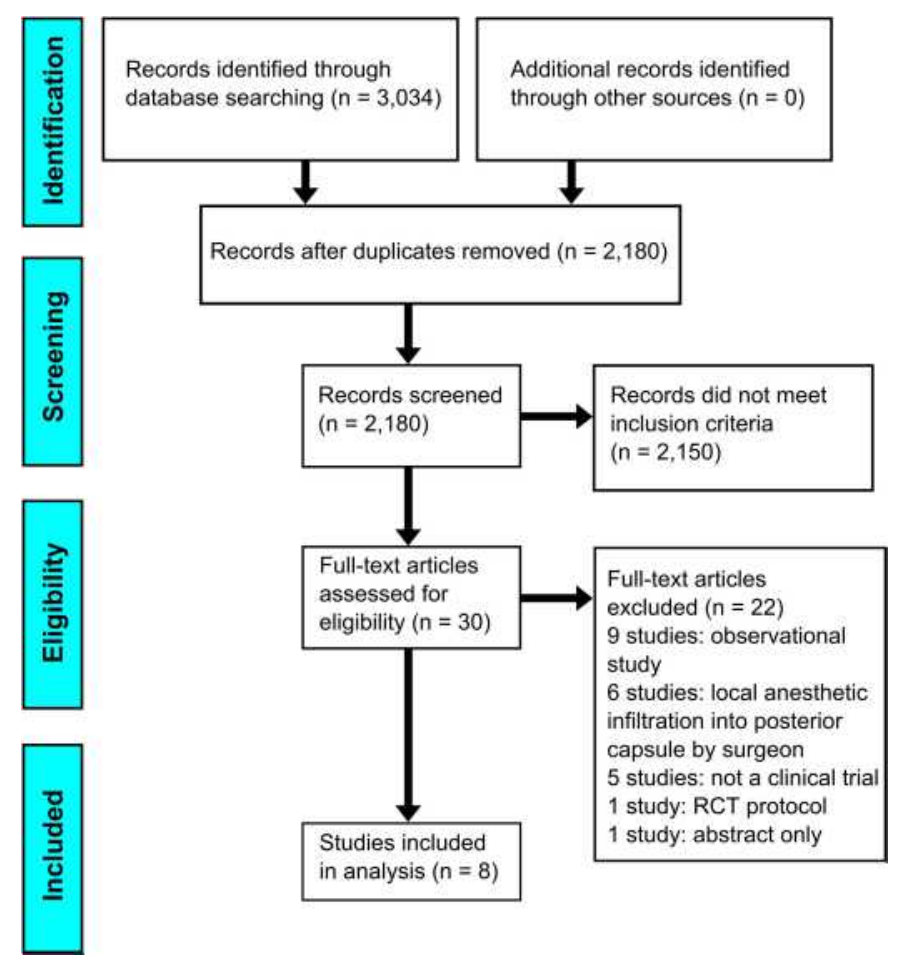

Figure I Flow chart of the study selection and inclusion process.

\section{Technique and Local Anesthetic Regimen} Three RCTs ${ }^{13,26,28}$ performed a proximal iPACK injection (distal femoral shaft), four RCTs ${ }^{27,29-31}$ performed a distal iPACK injection (femoral condyle), while one RCT $(\text { Kampitak et al })^{25}$ performed both variations. Local anesthetic dosing regimen for $\mathrm{PACK}$ blocks varied widely with three different types of local anesthetic medications utilized in included studies (ropivacaine, bupivacaine, and levobupivacaine). Five studies ${ }^{25,26,29-31}$ included epinephrine in the local anesthetic solution for the PPACK block. The most common formulation for iPACK was $0.25 \%$ levobupivacaine with epinephrine. ${ }^{25,29,31}$ Volume of injectate ranged from $20 \mathrm{~mL}$ to $25 \mathrm{~mL}$, with the most common being $20 \mathrm{~mL}$ in seven studies. ${ }^{25-31}$

\section{Primary Outcome \#I: Postoperative Opioid Consumption}

Data on postoperative opioid consumption either in the PACU or on postoperative day (POD) \#0 were reported in seven $\mathrm{RCTs}^{13,25-27,29-31}$ comprising a total of 606 patients (Table 3). Compared to controls, patients who received an iPACK block consumed less opioid in the PACU or POD \#0 in two ${ }^{13,30}$ of seven studies, consumed more opioid in one ${ }^{29}$ of seven studies, while the remaining studies $^{25-27,31}$ demonstrated no significant differences.
Data on postoperative opioid consumption on POD \#1 or 24-hour opioid consumption were available in seven $\mathrm{RCTs}^{13,25-27,29-31}$ comprising a total of 606 patients (Table $3)$. Compared to controls, patients who received an iPACK block consumed less opioid on POD \#1 or in a 24-hour period in one ${ }^{30}$ of seven studies, consumed more opioid in one $^{29}$ of seven studies, while the remaining studies $^{13,25-27,31}$ demonstrated no significant differences. Tak and colleagues ${ }^{28}$ analyzed cumulative OME at the time of discharge only (time not specified) and reported that the mean cumulative OME was higher in patients who received an $\mathrm{PACK}+\mathrm{ACB}$ block versus control patients receiving $\mathrm{cACB}$.

Given the heterogeneity in study arm treatments, meaningful comparisons are limited. However, three RCTs ${ }^{26-28}$ had similar study arm comparisons involving iPACK $+\mathrm{ACB}$ versus ACB only. In these RCTs, there was either no difference in postoperative OME consumption between study arms, ${ }^{26,27}$ or higher postoperative OME consumption in the $\mathrm{PACK}+\mathrm{ACB}$ arm. ${ }^{28}$

\section{Primary Outcome \#2: Postoperative Pain Score with Movement}

Patient report of postoperative pain with movement either in the PACU or on POD $\# 0$ were reported in five 
Table I GRADE Summary of Findings Table

\begin{tabular}{|c|c|c|c|}
\hline \multicolumn{4}{|c|}{$\begin{array}{l}\text { Patient or Population: Postope } \\
\text { Intervention: iPACK Cohort } \\
\text { Comparison: Control Cohort }\end{array}$} \\
\hline Outcomes & $\begin{array}{c}\text { Number of } \\
\text { Participants } \\
\text { (Studies) Follow Up }\end{array}$ & $\begin{array}{l}\text { Certainty of the Evidence } \\
\text { (GRADE) }\end{array}$ & Summary of Outcomes \\
\hline $\begin{array}{l}\text { Postoperative } \\
\text { opioid } \\
\text { consumption }\end{array}$ & 777 (8 RCTs) & $\oplus \oplus \oplus \bigcirc$ MODERATE ${ }^{a, b, c}$ & $\begin{array}{l}\text { In the PACU/POD\#0, iPACK arm had less opioid in 2/7 RCTs, } \\
\text { more opioid in I/7 RCTs, and no difference of opioid in 4/7 RCTs. } \\
\text { On POD\#I, iPACK arm has less opioid in I/7 RCTs, more opioid in } \\
\text { I/7 RCTs, and no different of opioid in 5/7 RCTs. }\end{array}$ \\
\hline $\begin{array}{l}\text { Postoperative } \\
\text { pain score with } \\
\text { movement }\end{array}$ & 658 (7 RCTs) & $\oplus \oplus \bigcirc \bigcirc$ LOW $^{\mathrm{a}, \mathrm{b}, \mathrm{c}, \mathrm{d}}$ & $\begin{array}{l}\text { In the PACU/POD\#0, iPACK arm had lower movement pain scores } \\
\text { in } 3 / 5 \text { RCTs, while } 2 / 5 \text { RCTs showed no difference. On POD\#I, } \\
\text { iPACK arm had lower movement pain scores in } 2 / 7 \text { RCTs, higher } \\
\text { movement pain scores in } 2 / 7 \text { RCTs, and no difference in movement } \\
\text { pain scores in } 3 / 7 \text { RCTs. }\end{array}$ \\
\hline $\begin{array}{l}\text { Postoperative } \\
\text { Pain Score at } \\
\text { Rest }\end{array}$ & 777 (8 RCTs) & $\oplus \oplus \bigcirc \bigcirc$ LOW $^{\mathrm{a}, \mathrm{b}, \mathrm{c}, \mathrm{d}}$ & $\begin{array}{l}\text { In the PACU/POD\#0, iPACK arm had lower rest pain score in } 3 / 6 \\
R C T s \text {, higher rest pain score in I/6 RCTs, and no difference in rest } \\
\text { pain score in } 2 / 6 \mathrm{RCTs} \text { compared to control. On POD\#I, iPACK } \\
\text { arm had lower rest pain score in } 2 / 8 \mathrm{RCTs} \text {, higher rest pain score in } \\
2 / 8 \mathrm{RCTs} \text {, and no difference in rest pain score in } 4 / 8 \mathrm{RCTs} \text {. }\end{array}$ \\
\hline $\begin{array}{l}\text { Hospital Length } \\
\text { of Stay }\end{array}$ & 353 (4 RCTs) & $\oplus \oplus \oplus \oplus \mathrm{HIGH}^{\mathrm{a}, \mathrm{b}}$ & $\begin{array}{l}\text { No difference in hospital length of stay was reported in } 3 / 4 \text { RCTs. } \\
\text { In Kampitak et al, the distal iPACK group had lower length of stay } \\
\text { compared to control. }\end{array}$ \\
\hline Gait Distance & 374 (4 RCTs) & $\oplus \oplus \oplus \oplus \mathrm{HIGH}^{\mathrm{a}, \mathrm{b}}$ & No difference in gait distance was reported in $4 / 4$ RCTs. \\
\hline $\begin{array}{l}\text { Knee Range of } \\
\text { Motion }\end{array}$ & 332 (4 RCTs) & $\oplus \oplus \oplus \bigcirc$ MODERATE ${ }^{\mathrm{a}, \mathrm{b}, \mathrm{e}}$ & $\begin{array}{l}\text { No difference in knee ROM was reported in } 3 / 4 \text { RCTs. Compared to } \\
\text { the control, knee flexion was decreased on POD\#0 and extension was } \\
\text { decreased on POD\#I in the iPACK arm in I/4 RCTs. }\end{array}$ \\
\hline $\begin{array}{l}\text { Patient } \\
\text { Satisfaction }\end{array}$ & 303 (3 RCTs) & $\oplus \oplus \oplus \bigcirc$ MODERATE ${ }^{a, b, f}$ & $\begin{array}{l}\text { No difference in patient satisfaction was reported between study } \\
\text { arms in } 2 / 3 \text { RCTs. In one RCT, patient satisfaction was increased in } \\
\text { the PACU and on POD\#I. }\end{array}$ \\
\hline
\end{tabular}

Notes: GRADE Working Group grades of evidence. High certainty: We are very confident that the true effect lies close to that of the estimate of the effect. Moderate certainty: We are moderately confident in the effect estimate: The true effect is likely to be close to the estimate of the effect, but there is a possibility that it is substantially different. Low certainty: Our confidence in the effect estimate is limited: The true effect may be substantially different from the estimate of the effect. Very low certainty: We have very little confidence in the effect estimate: The true effect is likely to be substantially different from the estimate of effect. Explanations: ${ }^{\text {In }}$ some RCTs, anesthesiologist (involved in study) was not blinded to study arms. ${ }^{b}$ Other sources of bias included no true control group without a posterior knee block, unequal dose of local anesthetic between study arms, inclusion of chronic pain patients in some studies, and heterogeneity in injection location of iPACK. 'Some studies showed outcomes favoring iPACK, some studies showed no association, and some studies showed worse outcomes from iPACK. 'Effect estimates and direction of association between treatment arms were widely variable among included RCTs for the specified outcome. ${ }^{e}$ One study showed outcome not favoring iPACK, while other studies showed no association. ${ }^{f}$ One study showed outcome favoring iPACK, while other studies showed no association.

$\mathrm{RCTs}^{13,26,29-31}$ comprising a total of 389 patients (Table 4). Compared to controls, patients who received an iPACK block reported lower pain scores with movement in the PACU or on POD \#0 in three ${ }^{13,30,31}$ of five studies, while the remaining studies ${ }^{26,29}$ demonstrated no significant differences.

Patient report of postoperative pain with movement either on POD \#1 or after a 24 -hour period were available in seven RCTs $^{13,25,26,28-31}$ comprising a total of 658 patients (Table 4).
Compared to controls, patients who received an iPACK block reported lower pain scores with movement on POD \#1 or in a 24-hour period in two ${ }^{13,31}$ of seven studies, reported higher pain scores with movement in two ${ }^{25,28}$ of seven studies, while the remaining studies ${ }^{26,29,30}$ demonstrated no significant differences. Of note, while Vichainarong et $\mathrm{al}^{31}$ reported lower pain scores with movement favoring the iPACK arm in the PACU and on POD\#1, they deemed the statistical difference as not being clinically significant. 


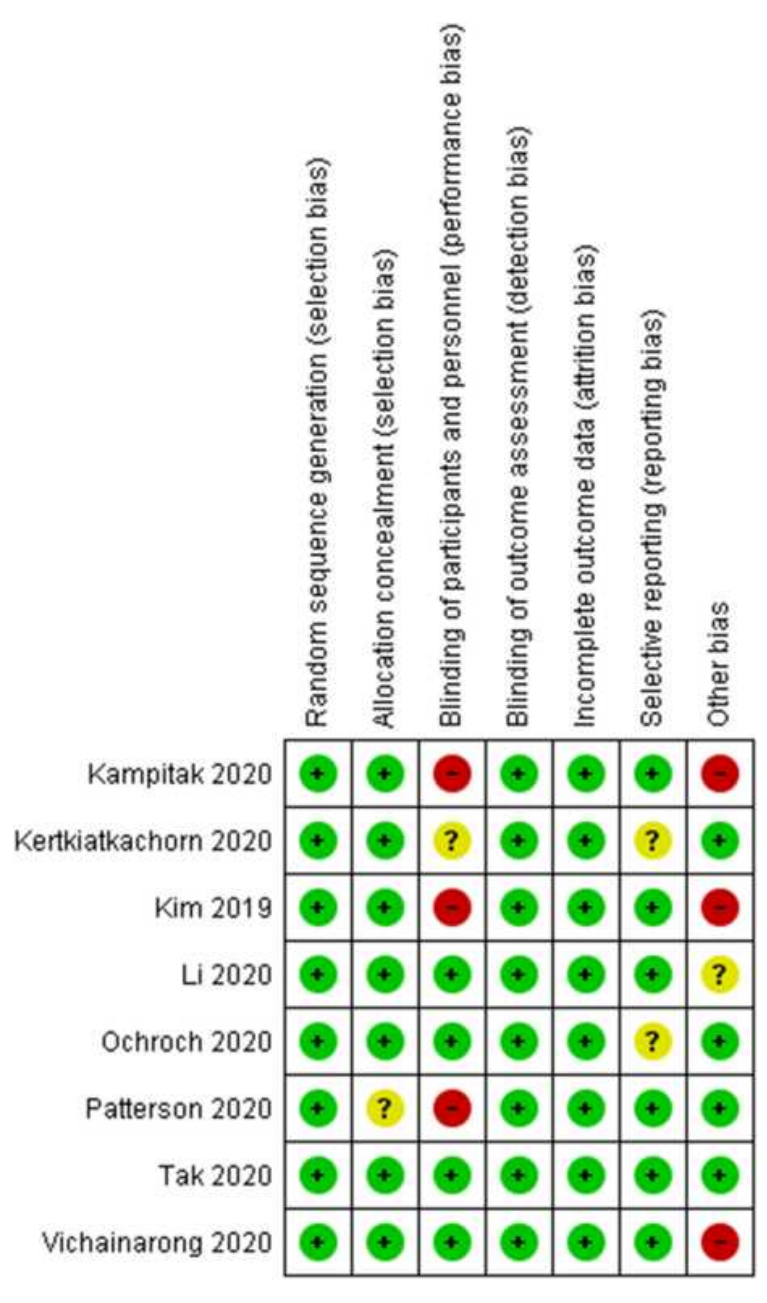

Figure 2 Risk of bias summary for randomized controlled trials.

\section{Secondary Outcome Measures}

The Supplemental File presents a summary of secondary outcome measures. Inconsistency of the findings and differing estimates of the treatment effect were observed across several outcomes including pain at rest, patient satisfaction, hospital length of stay, and knee ROM. Consistency of the study findings was observed in gait distance, which demonstrated no significant associations between treatment arms. There were no significant differences in any adverse event. The power of included studies was insufficient to draw meaningful data and conclusions for adverse events. Kampitak and colleagues ${ }^{25}$ identified no difference in duration of peroneal nerve blockade between study arms (tibial nerve block versus proximal iPACK block versus distal iPACK block). They identified greater duration of tibial nerve blockade in the tibial nerve block arm versus the proximal iPACK arm, but no difference when compared to the distal iPACK arm. ${ }^{25}$

\section{Discussion}

\section{Main Findings from Qualitative Synthesis}

This systematic review examined the potential added analgesic benefit for inclusion of iPACK block following primary TKA. Our analysis revealed that patients who received an iPACK block with a peripheral nerve block generally had similar postoperative opioid consumption in the PACU and up to 24 hours postoperatively after TKA compared to those who did not receive an iPACK block (moderate-quality GRADE evidence). When analyzing RCTs with similar study arms, the comparison of iPACK $+\mathrm{ACB}$ versus $\mathrm{ACB}$ did not reveal any difference in postoperative OME consumption between study arms, and surprisingly, one study reported a higher, albeit not clinically significant, postoperative OME consumption in the iPACK $+\mathrm{ACB}$ arm. ${ }^{28}$ Congruent with this, the majority of eligible studies reported no difference in patient satisfaction, hospital LOS, gait distance, and knee ROM. Widely differing estimates of treatment effect were observed for pain scores with movement. While the majority of eligible RCTs reported an improvement in pain with movement in the PACU or on POD\#0, most eligible studies failed to show any difference on POD\#1 (low-quality GRADE evidence).

\section{Does the Addition of iPACK Block Benefit Patients Undergoing TKA Compared to Current Enhanced Recovery Pathways?}

Enhanced recovery after Surgery (ERAS) pathways have established that regional anesthesia, specifically neuraxial anesthesia, are integral modalities to decrease opioid exposure and improve analgesic outcomes perioperatively. ${ }^{32,33}$ While our qualitative synthesis of the included RCTs is constrained by the limitations discussed below, the findings were unexpected as studies have often demonstrated the need for posterior knee coverage after TKA. Even after patients receive a successful femoral nerve block, 60-90\% require further treatment for postoperative knee pain, presumably due to inadequate coverage. ${ }^{34-36}$ Although prior RCTs $^{37-39}$ have demonstrated that the addition of sciatic nerve blockade to femoral nerve blockade decreased pain scores and postoperative opioid requirements, the profound lower extremity paralysis makes this combination unfavorable to both early mobilization and postoperative neurologic 


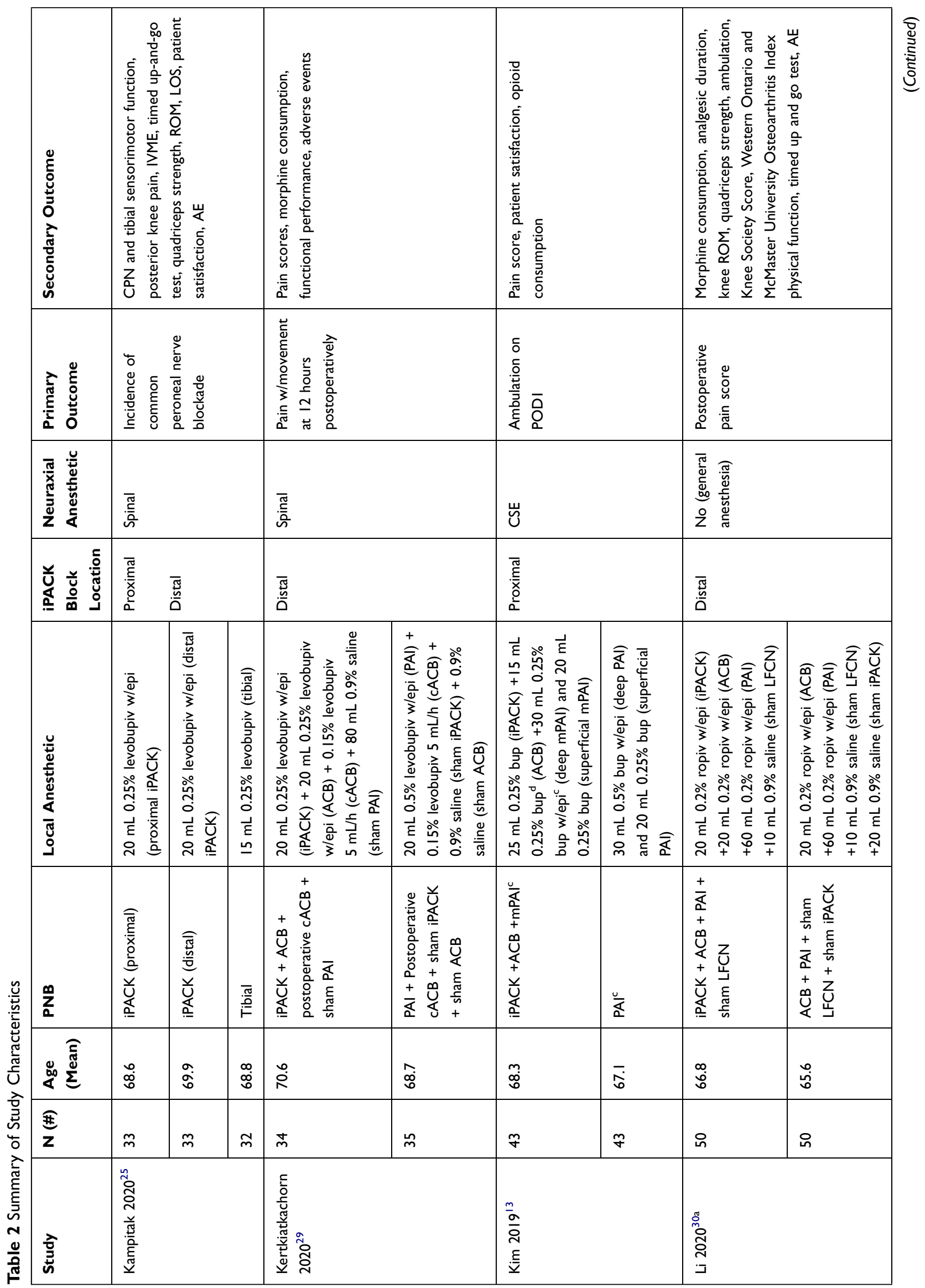




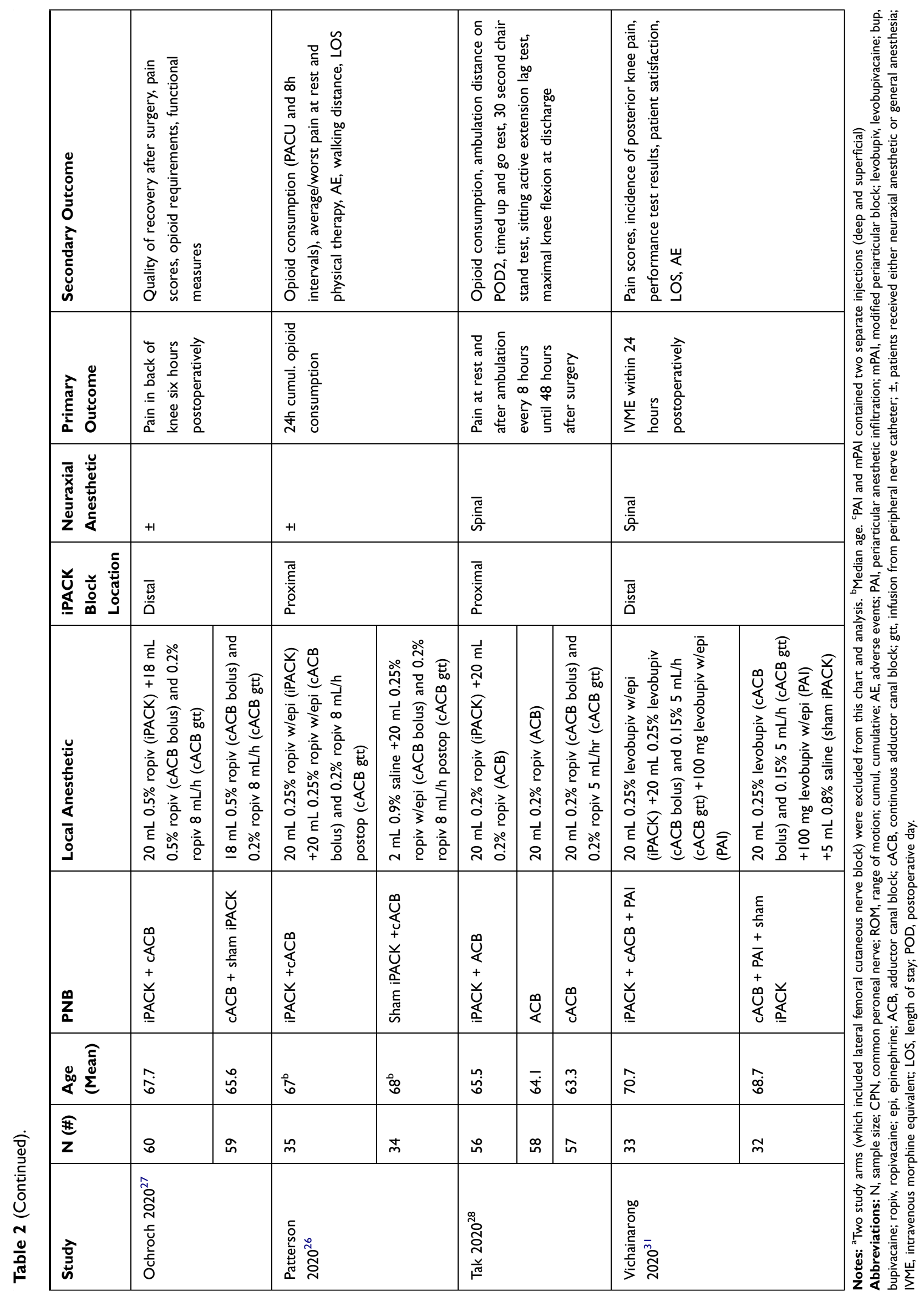


Table 3 Summary of Postoperative Opioid Consumption in First 24 Hours

\begin{tabular}{|c|c|}
\hline Study & Summary of Result \\
\hline \multicolumn{2}{|c|}{ Studies with Significant Association Favoring iPACK } \\
\hline $\operatorname{Kim} 2019^{13}$ & $\begin{array}{l}\text { Patients receiving iPACK }+A C B+m P A I \text { had lower mean OME in PACU compared to control arm receiving PAI only (control } \\
27.7 \pm 21.7 \mathrm{mg} \text {, iPACK } 14.9 \pm 19.4 \mathrm{mg}, \mathrm{p}=0.005 \text { ), but no differences of cumulative opioid consumption from } 0-24 \text { hours }\end{array}$ \\
\hline Li $2020^{30}$ & $\begin{array}{l}\text { Patients receiving iPACK }+A C B+P A I \text { had lower mean } O M E \text { in the first } 24 \text { hours compared to the control arm receiving } A C B \\
+P A I \text { (control } 16.00 \pm 7.82 \text {, iPACK } 10.8 \pm 8.77, p<0.001 \text { ) }\end{array}$ \\
\hline \multicolumn{2}{|c|}{ Studies with Significant Association Not Favoring iPACK } \\
\hline $\begin{array}{l}\text { Kertkiatkachorn } \\
2020^{29}\end{array}$ & $\begin{array}{l}\text { Median cumulative IVME was higher in patients receiving iPACK+ACB+cACB compared to patients receiving } c A C B+P A I \text { at } \\
12 \text { hours }(0[0-2] \mathrm{mg} \text { vs } 0[0-0] \mathrm{mg}, \mathrm{p}=0.004) \text { and } 24 \text { hours postoperatively }(2[0-4] \mathrm{mg} \text { vs } 0[0-0] \mathrm{mg}, \mathrm{p}=0.002)\end{array}$ \\
\hline Tak $2020^{28}$ & $\begin{array}{l}\text { Mean cumulative OME at time of discharge was higher in patients receiving iPACK+ACB }(31.110 \pm 5.649) \text { as well as patients } \\
\text { receiving } A C B \text { only }(33.520 \pm 7.052) \text { compared to patients receiving } C A C B \text { only }(30.110 \pm 5.257, p=0.011)\end{array}$ \\
\hline \multicolumn{2}{|c|}{ Studies with No Significant Association } \\
\hline Kampitak $2020^{25}$ & $\begin{array}{l}\text { Median cumulative IVME was } 2(2-2) \text { for tibial group, } 2(0-2) \mathrm{mg} \text { for proximal iPACK group, and } 2(2-2) \mathrm{mg} \text { for distal iPACK } \\
\text { group at both } 12 \text { hours and } 24 \text { hours postoperatively. No significant difference was detected for both comparisons }\end{array}$ \\
\hline Patterson $2020^{26}$ & $\begin{array}{l}\text { There were no significant differences in median cumulative OME between the iPACK }+c A C B \text { arm and control arm (cACB } \\
\text { only) in PACU (control } 23[0-38] \mathrm{mg} \text {, iPACK } 23 \text { [15-29] mg, } \mathrm{p}=0.7928 \text { ) and up to } 24 \text { hours (control } 79 \text { [58-123] mg, iPACK } \\
90[60-128] \mathrm{mg}, \mathrm{p}=0.7456)\end{array}$ \\
\hline Ochroch $2020^{27}$ & $\begin{array}{l}\text { There were no significant differences in mean cumulative OME between iPACK }+c A C B \text { arm and control arm ( } A \text { ACB only) at } \\
0-12 \text { hours (control } 30[15-45] \mathrm{mg} \text {, iPACK } 30[15-45] \mathrm{mg}, \mathrm{p}=0.59 \text { ) and at } 12-24 \text { hours postoperatively (control } 15[7.5-30] \text {, } \\
\text { iPACK } 15[7.5-30], \mathrm{P}=0.37 \text { ) }\end{array}$ \\
\hline $\begin{array}{l}\text { Vichainarong } \\
2020^{31}\end{array}$ & $\begin{array}{l}\text { There were no significant differences in mean cumulative IVME between iPACK }+c A C B+P A I \text { arm and control arm (cACB } \\
+P A I) \text { at } 12 \text { hours (control } 0.4 \pm I \mathrm{mg} \text {, iPACK } 0.1 \pm 0.5 \mathrm{mg}, \mathrm{p}=0 . \mathrm{I} \text { ) and } 24 \text { hours postoperative (control } 1.3 \pm 1.9 \text {, iPACK } 0.6 \\
\pm \mathrm{I} .3, \mathrm{p}=0.08 \text { ) }\end{array}$ \\
\hline
\end{tabular}

Notes: Postoperative opioid consumption in PACU and up to 24 hours postoperatively are documented and presented in the table. When reported and available in study, mean ( \pm standard deviation) and median (25\%-75\%ile interquartile range) were provided.

Abbreviations: IVME, IV morphine equivalents; OME, oral morphine equivalents; NRS, numerical rating scale; POD, postoperative day; FNC, femoral nerve catheter; ACB, adductor canal block; CACB, continuous adductor canal block; mPAl, modified arthroplasty block.

surveillance. The introduction of the iPACK block in combination with an ACB has been speculated to be an effective pain control combination that also minimizes the potential for muscle weakness. However, results of this review fail to support the benefit of adding an iPACK block for the purposes of opioid-sparing analgesia. The authors acknowledge that confounders within the studies included in this review must be taken into account, such as flaws in study design or execution of other treatments that may provide for posterior knee coverage such as sciatic blocks or PAI in some of the experimental arms. ${ }^{13,40}$ When assessing only RCTs with treatment arms comparing $\mathrm{PACK}+\mathrm{ACB}$ vs $\mathrm{PAI}$ $+\mathrm{ACB}$, two $\mathrm{RCTs}^{29,31}$ reported no clinically significant difference in opioid consumption or pain scores with movement. While this may indicate that PAI targets similar nerves that overlap with the iPACK coverage, additional objective studies, such as cadaver studies comparing injectate spread, are warranted.
Interestingly, five $\mathrm{e}^{26-29,31}$ of six studies that either did not reveal an association or revealed higher postoperative OME in the iPACK cohort contained a control arm that delivered cACB. A similar observation was also noted in the variable of pain with movement, where three ${ }^{26,28,29}$ of four studies either reported no difference or worse pain scores in the iPACK arm containing a control arm that delivered cACB. This may reflect a component of continuous local anesthetic spread from the adductor canal to the genicular branches of the posterior obturator nerve as well as the popliteal plexus, which has been demonstrated in cadaveric studies ${ }^{41}$ and in patients undergoing TKA. ${ }^{42}$ This spread would potentially negate the benefit of the iPACK block for posterior compartment analgesia. We also noted that Tak and colleagues ${ }^{28}$ reported a higher cumulative OME in patients who received an $\mathrm{PACK}+\mathrm{ACB}$ block versus control patients who received cACB at discharge only (time not specified); this outcome may be due to the limited duration of both single-injection 
Table 4 Summary of Findings for Assessment of Postoperative Pain Score with Movement in First 24 Hours

\begin{tabular}{|c|c|}
\hline Study & Summary of Result \\
\hline \multicolumn{2}{|c|}{ Studies with Significant Association Favoring iPACK } \\
\hline $\operatorname{Kim} 2019^{13}$ & 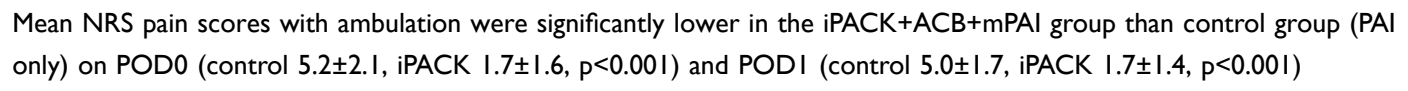 \\
\hline Li $2020^{30}$ & $\begin{array}{l}\text { Mean VAS pain scores with activity were significantly lower in the iPACK+ACB+PAI arm than the control group }(A C B+P A I) \\
\text { at } 12 \text { hours postoperatively (control } 5.52 \pm 0.86 \text {, iPACK } 5.23 \pm 0.98, p=0.002) \text {, but there was no difference at } 24 \text { hours } \\
\text { postoperative (control } 4.96 \pm 0.78 \text {, iPACK } 4.78 \pm 1.02, p=0.312 \text { ) }\end{array}$ \\
\hline $\begin{array}{l}\text { Vichainarong } \\
2020^{31}\end{array}$ & $\begin{array}{l}\text { Mean NRS pain scores with movement were significantly lower in the iPACK }+c A C B+P A I \text { than the control group }(P A I+c A C B) \\
\text { in the PACU (control } 0 \pm 0 \text {, iPACK } 0.1 \pm 0.1, P=0.0 I \text { ) and at } 24 \text { hours postoperatively (control } 2.2 \pm 0.3, i P A C K 2 . I \pm 0.3 \text {, } \\
P=0.00 I \text { ); however, the authors deemed this statistical difference as not being clinically significant }\end{array}$ \\
\hline \multicolumn{2}{|c|}{ Studies with Significant Association Not Favoring iPACK } \\
\hline Kampitak $2020^{25}$ & $\begin{array}{l}\text { NRS pain scores with movement were significantly higher in the proximal iPACK group than in the tibial nerve block group } \\
\text { and distal iPACK group }(p=0.001) \text { at } 24 \text { hours postoperatively }\end{array}$ \\
\hline Tak $2020^{28}$ & $\begin{array}{l}\text { Mean VAS pain scores after ambulation were significantly higher in the iPACK }+A C B \text { arm }(4.536 \pm 0.503) \text { and } A C B \text { arm }(5.845 \\
\pm 0.790) \text { compared to the } C A C B \text { arm }(3.702 \pm 0.597, p<0.001) \text { at PODI and } 2\end{array}$ \\
\hline \multicolumn{2}{|c|}{ Studies Showing no Difference } \\
\hline $\begin{array}{l}\text { Kertkiatkachorn } \\
2020^{29}\end{array}$ & $\begin{array}{l}\text { There was no difference in mean anterior knee and posterior knee VAS pain scores with movement between patients } \\
\text { receiving iPACK }+A C B+C A C B \text { versus control patients receiving } P A I+C A C B \text { in the } P A C U \text {, at } 12 \text { hours, and } 24 \text { hours } \\
\text { postoperatively }\end{array}$ \\
\hline Patterson $2020^{26}$ & $\begin{array}{l}\text { There was no difference in median VAS pain score with movement/physical therapy between patients receiving iPACK }+c A C B \\
\text { and control patients receiving } c A C B \text { only in the PACU (Control } 5[3-7] \text {, iPACK } 4[2-6], p=0.2080 \text { ) and on PODI (Control } 5 \\
{[4-7], \text { iPACK } 5[1-7], p=0.1488 \text { ) }}\end{array}$ \\
\hline
\end{tabular}

Notes: Postoperative pain scores with movement in PACU and up to 24 hours are documented and presented in the table. When reported and available in study, mean ( \pm standard deviation) and median (25\%-75\%ile interquartile range) were provided.

Abbreviations: NRS, numerical rating scale; VAS, visual analog scale; POD, postoperative day; POD, postoperative day; FNC, femoral nerve catheter; ACB, adductor canal block; $\mathrm{CACB}$, continuous adductor canal block; mPAl, modified arthroplasty block.

iPACK and single-injection ACB versus extended duration provided by a continuous ACB catheter. ${ }^{43}$

Although not linked to reductions in the outcomes of opioid consumption and pain scores with movement in most of the evaluated studies, it is still plausible that iPACK block use provides advantages not thoroughly explored in the existing TKA literature. The iPACK block has the ability to anesthetize articular nerves that transmit posterior knee pain while limiting blockade of motor fibers. This is consistent with cadaveric studies demonstrating local anesthetic spread throughout the popliteal fossa, but without localization to the proximal segment of the sciatic nerve. ${ }^{44}$ Further, it is unknown if iPACK block may be an advantageous addition for patients with expected higher postoperative pain intensity and analgesic requirements.

The need for a feasible postoperative rescue block for posterior knee pain after TKA is commonly brought up in recovery pathway discussions. An iPACK block may be challenging to perform in the postoperative setting, when the knee joint can be covered with dressings that limit access or surgeon preference to avoid violation of the operated knee joint is recognized. In this scenario, a popliteal plexus block may serve as a suitable rescue regional block as described by Runge and colleagues. ${ }^{45}$

\section{Which iPACK Block Technique is Preferable?}

Two iPACK block locations have been described in eligible studies: proximal (injecting at the distal femoral shaft approximately one to two fingerbreadths above the patellar base ${ }^{44}$ and distal (injecting at the upper area of the femoral condyle) ${ }^{46,47}$ A cadaveric study ${ }^{12}$ comparing the two approaches demonstrated that the proximal approach promoted greater anteromedial dye spread, whereas the distal approach had more anterolateral spread. The 
proximal approach would more consistently involve the superior medial genicular nerve, while the distal approach would more consistently involve the superior lateral genicular nerve as well as the anterior branch of the common fibular nerve. ${ }^{12}$

Of the included RCTs, only one directly compared outcomes based on needle site entry. ${ }^{25}$ Kampitak and colleagues ${ }^{25}$ observed that patients who received the distal iPACK block reported improved preservation of motor function compared to those receiving proximal iPACK and tibial blocks. Furthermore, posterior knee pain scores were similar in the distal iPACK and tibial nerve groups, but significantly worse in the proximal iPACK group. Although cadaveric studies have reported that the proximal iPACK injection site may be favorable due to a decreased risk of inadvertent intra-articular injection, ${ }^{44}$ this was not reported by Kampitak and colleagues. Among the remaining included RCTs, approximately half performed the proximal approach and half performed the distal approach, making meaningful comparisons unable to be performed. There is a need for comparative studies based on iPACK block location.

\section{What are Potential Adverse Effects from the iPACK Block?}

There were no significant differences in any adverse events, including allergic reactions, nerve injury, nausea/ vomiting, and respiratory depression between study arms. Admittedly, the limited sample size of this review would not allow for certainty for such low-frequency complications. The proximity of the popliteal artery and its perforator branches introduces the risk of intravascular injection. The potential for local anesthetic systemic toxicity (LAST) exists in any regional technique. The dose of bupivacaine in the interventional arm in one included study $^{13}$ and the dose of ropivacaine in the intervention arm in another study ${ }^{30}$ exceeded many published safe dosing ranges for local anesthetics. ${ }^{48,49}$ Future studies should maintain conservative dosing strategies as LAST events, although rare, are challenging to detect in small study populations. ${ }^{48,49}$

\section{Limitations}

This review is limited by the quantity and quality of available evidence. Sample sizes were small across included studies, although this would not be atypical of trials performed to evaluate novel regional anesthesia techniques. There was considerable heterogeneity among the included studies in terms of exposure variables and outcomes assessed. Confounding factors included unequal doses of local anesthetic administered between study arms, pre-operative opioid use, no standardization in multimodal analgesic management, inconsistency in patients receiving neuraxial anesthesia between study arms, inability to blind anesthesiologists performing the regional blocks, and lack of control groups administering pre-emptive oral multimodal analgesia without any regional analgesia alone. Registration conflicts were present in two included trials; ${ }^{26,30}$ Patterson et $\mathrm{al}^{26}$ performed a retrospective registration, and $\mathrm{Li}$ et $\mathrm{al}^{30}$ increased the study sample size from 125 to 200 participants and created different study groups. Kim et $\mathrm{al}^{13}$ compared $\mathrm{iPACK}+\mathrm{ACB}+\mathrm{PAI}$ (intervention arm) versus PAI (control arm), and consequently this comparison may not allow differentiation of the analgesic treatment effects of $\mathrm{PACK}$ or $\mathrm{ACB}$ since both were not present as controls. Finally, systematic reviews are generally poor at identifying rare side effects due to clinical and methodological heterogeneity and inconsistency in reporting complications. ${ }^{50}$

\section{Recommendations for Future Research}

While one study included posterior knee blocks in the control cohort, specifically tibial nerve block, ${ }^{25}$ further research and separate subgroup analysis would be necessary to directly compare iPACK block versus other posterior knee blocks (eg proximal sciatic nerve block, selective tibial nerve block). Larger-scale RCTs are warranted and should focus on more objective measures of pain, including metrics of physical function, quality of recovery, and opioid consumption beyond the immediate perioperative period. Future studies should investigate outcomes over a longer time period after the first postoperative day. This may help determine if decreased pain immediately postoperatively after an iPACK block may be associated with improved analgesia further out from surgery. Doseresponse studies are warranted to identify the minimal effective concentration and volume for iPACK blocks. Future studies should compare the duration of analgesia, efficacy, cost-effectiveness, functional outcomes, and healthcare utilization between ultrasound-guided iPACK blocks versus other posterior knee blocks and surgeon-administered posterior knee capsule local infiltration analgesia. Finally, trials should also consider expanding inclusion criteria for the pediatric 
population $^{51}$ and other types of knee surgeries besides TKA, including knee ligament repair or reconstruction, meniscus repair, and unicompartmental partial knee replacements.

In conclusion, this review provides a foundation for current evidence on iPACK efficacy while acknowledging gaps in knowledge. While the existing literature does not presently support routine use of iPACK block, future RCTs are warranted to further evaluate clinical utility in specific TKA populations, to optimize local anesthetic dosing and volume, and to provide further safety evidence prior to more widespread implementation of iPACK block use for TKA.

\section{Author Contributions}

Ryan S. D'Souza MD: This author helped perform background of research, study conception and design, search strategy and study selection, assessment of bias and quality, statistical analysis, data collection, generation of figures, analysis and interpretation of data, and drafted manuscript.

Brendan J. Langford MD: This author helped perform background of research, search strategy and study selection, assessment of bias and quality, data collection, and revised manuscript.

David A. Olsen MD: This author helped perform background of research, analysis and interpretation of data, drafted manuscript, and revised the manuscript critically for intellectual content and gave final approval of the manuscript.

Rebecca L. Johnson MD: This author helped with study conception and design, background of research, analysis and interpretation of data, drafted manuscript, and revised the manuscript critically for intellectual content and gave final approval of the manuscript.

All authors made substantial contributions to conception and design, acquisition of data, or analysis and interpretation of data; took part in drafting the article or revising it critically for important intellectual content; agreed to submit to the current journal; gave final approval for the version to be published; and agreed to be accountable for all aspects of the work.

\section{Funding}

There is no funding to report.

\section{Disclosure}

The authors reported no conflicts of interest for this work.

\section{References}

1. Feng JE, Novikov D, Anoushiravani AA, Schwarzkopf R. Total knee arthroplasty: improving outcomes with a multidisciplinary approach. J Multidiscip Healthc. 2018;11:63-73. doi:10.2147/JMDH.S140550

2. Society KK. Guidelines for the management of postoperative pain after total knee arthroplasty. Knee Surg Relat Res. 2012;24 (4):201-207. doi:10.5792/ksrr.2012.24.4.201

3. Maheshwari AV, Blum YC, Shekhar L, Ranawat AS, Ranawat CS. Multimodal pain management after total hip and knee arthroplasty at the Ranawat Orthopaedic Center. Clin Orthop Relat Res. 2009;467 (6):1418-1423. doi:10.1007/s11999-009-0728-7

4. Sinatra RS, Torres J, Bustos AM. Pain management after major orthopaedic surgery: current strategies and new concepts. $J \mathrm{Am}$ Acad Orthop Surg. 2002;10(2):117-129. doi:10.5435/00124635200203000-00007

5. Gerbershagen HJ, Aduckathil S, van Wijck AJ, Peelen LM, Kalkman CJ, Meissner W. Pain intensity on the first day after surgery: a prospective cohort study comparing 179 surgical procedures. Anesthesiology. 2013;118(4):934-944. doi:10.1097/ALN.0b013e318 $28866 \mathrm{~b} 3$

6. Kopp SL, Børglum J, Buvanendran A, et al. Anesthesia and analgesia practice pathway options for total knee arthroplasty: an evidence-based review by the American and European societies of regional anesthesia and pain medicine. Reg Anesth Pain Med. 2017;42(6):683-697. doi:10.1097/AAP.0000000000000673

7. Terkawi AS, Mavridis D, Sessler DI, et al. Pain management modalities after total knee arthroplasty: a network meta-analysis of 170 Randomized Controlled Trials. Anesthesiology. 2017;126 (5):923-937. doi:10.1097/ALN.0000000000001607

8. Kehlet H, Andersen L. Local infiltration analgesia in joint replacement: the evidence and recommendations for clinical practice. Acta Anaesthesiol Scand. 2011;55(7):778-784. doi:10.1111/j.1399-6576. 2011.02429.x

9. Orduña Valls JM, Vallejo R, López Pais P, et al. Anatomic and ultrasonographic evaluation of the knee sensory innervation: a cadaveric study to determine anatomic targets in the treatment of chronic knee pain. Reg Anesth Pain Med. 2017;42(1):90-98. doi:10.1097/AAP.0000000000000516

10. Laoruengthana A, Rattanaprichavej P, Rasamimongkol S, Galassi M. Anterior vs posterior periarticular multimodal drug injections: a randomized, controlled trial in simultaneous bilateral total knee arthroplasty. J Arthroplasty. 2017;32(7):2100-2104. doi:10.1016/j. arth.2017.02.033

11. Ilfeld BM, McCartney CJL. Searching for the optimal pain management technique after knee arthroplasty: analgesia is just the tip of the iceberg. Anesthesiology. 2017;126(5):768-770. doi:10.1097/ALN.00 00000000001608

12. Tran J, Giron Arango L, Peng P, Sinha SK, Agur A, Chan V. Evaluation of the iPACK block injectate spread: a cadaveric study. Reg Anesth Pain Med. 2019;44(7):689-694. doi:10.1136/rapm-2018100355

13. Kim DH, Beathe JC, Lin Y, et al. Addition of infiltration between the popliteal artery and the capsule of the posterior knee and adductor canal block to periarticular injection enhances postoperative pain control in total knee arthroplasty: a Randomized Controlled Trial. Anesth Analg. 2019;129(2):526-535. doi:10.1213/ANE.0000000000 003794

14. Kardash KJ, Noel GP. The SPANK block: a selective sensory, single-injection solution for posterior pain after total knee arthroplasty. Reg Anesth Pain Med. 2016;41(1):118-119. doi:10.1097/AAP.0000000000000330

15. Page MJ, Shamseer L, Tricco AC. Registration of systematic reviews in PROSPERO: 30,000 records and counting. Syst Rev. 2018;7(1):32. doi:10.1186/s13643-018-0699-4 
16. Liberati A, Altman DG, Tetzlaff J, et al. The PRISMA statement for reporting systematic reviews and meta-analyses of studies that evaluate health care interventions: explanation and elaboration. $J$ Clin Epidemiol. 2009;62(10):e1-34. doi:10.1016/j.jclinepi.2009.06.006

17. Dalury DF. Periarticular injection technique to enhance pain relief after knee arthroplasty. JBJS Essent Surg Tech. 2014;4(2):e7. doi:10.2106/JBJS.ST.N.00001

18. Higgins JP, Altman DG, Gøtzsche PC, et al. The Cochrane Collaboration's tool for assessing risk of bias in randomised trials. BMJ. 2011;343(oct18 2):d5928. doi:10.1136/bmj.d5928

19. Kavanagh BP. The GRADE system for rating clinical guidelines. PLoS Med. 2009;6(9):e1000094. doi:10.1371/journal.pmed.1000094

20. Guyatt GH, Oxman AD, Vist G, et al. GRADE guidelines: 4. Rating the quality of evidence-study limitations (risk of bias). $J$ Clin Epidemiol. 2011;64(4):407-415. doi:10.1016/j.jclinepi.2010.07.017

21. Guyatt GH, Oxman AD, Kunz R, et al. GRADE guidelines: 7. Rating the quality of evidence-inconsistency. $J$ Clin Epidemiol. 2011;64 (12):1294-1302. doi:10.1016/j.jclinepi.2011.03.017

22. Guyatt GH, Oxman AD, Kunz R, et al. GRADE guidelines: 8. Rating the quality of evidence-indirectness. J Clin Epidemiol. 2011;64 (12):1303-1310. doi:10.1016/j.jclinepi.2011.04.014

23. Guyatt GH, Oxman AD, Kunz R, et al. GRADE guidelines 6. Rating the quality of evidence-imprecision. J Clin Epidemiol. 2011;64 (12):1283-1293. doi:10.1016/j.jclinepi.2011.01.012

24. Guyatt GH, Oxman AD, Montori V, et al. GRADE guidelines: 5 . Rating the quality of evidence-publication bias. $J$ Clin Epidemiol. 2011;64(12):1277-1282. doi:10.1016/j.jclinepi.2011. 01.011

25. Kampitak W, Tanavalee A, Ngarmukos S, Tantavisut S. Motor-sparing effect of iPACK (interspace between the popliteal artery and capsule of the posterior knee) block versus tibial nerve block after total knee arthroplasty: a randomized controlled trial. Reg Anesth Pain Med. 2020;45(4):267-276. doi:10.1136/rapm-2019-100895

26. Patterson ME, Vitter J, Bland K, Nossaman BD, Thomas LC, Chimento GF. The Effect of the IPACK block on pain after primary TKA: a double-blinded, prospective, randomized Trial. $J$ Arthroplasty. 2020;35(6):S173-S177. doi:10.1016/j.arth.2020.01.014

27. Ochroch J, Qi V, Badiola I, et al. Analgesic efficacy of adding the IPACK block to a multimodal analgesia protocol for primary total knee arthroplasty. Reg Anesth Pain Med. 2020;45(10):799-804. doi:10.1136/rapm-2020-101558

28. Tak R, Gurava Reddy AV, Jhakotia K, Karumuri K, Sankineani SR. Continuous adductor canal block is superior to adductor canal block alone or adductor canal block combined with IPACK block (interspace between the popliteal artery and the posterior capsule of knee) in postoperative analgesia and ambulation following total knee arthroplasty: randomized control trial. Musculoskelet Surg. 2020. doi:10.1007/s12306-020-00682-8

29. Kertkiatkachorn W, Kampitak W, Tanavalee A, Ngarmukos S. Adductor canal block combined with iPACK (Interspace Between the Popliteal Artery and the Capsule of the Posterior Knee) block vs periarticular injection for analgesia after total knee arthroplasty: a Randomized Noninferiority Trial. J Arthroplasty. 2020;36(1):122129.e1. doi:10.1016/j.arth.2020.06.086

30. Li D, Alqwbani M, Wang Q, Liao R, Yang J, Kang P. Efficacy of adductor canal block combined with additional analgesic methods for postoperative analgesia in total knee arthroplasty: a prospective, double-blind, randomized controlled Study.. J Arthroplasty. 2020;35 (12):3554-3562. doi:10.1016/j.arth.2020.06.060

31. Vichainarong C, Kampitak W, Tanavalee A, Ngarmukos S, Songborassamee N. Analgesic efficacy of infiltration between the popliteal artery and capsule of the knee (iPACK) block added to local infiltration analgesia and continuous adductor canal block after total knee arthroplasty: a randomized clinical trial. Reg Anesth Pain Med. 2020;45(11):872-879. doi:10.1136/rapm-2020101396
32. Ljungqvist $\mathrm{O}$, Scott M, Fearon KC. Enhanced recovery after surgery: a Review. JAMA Surg. 2017;152(3):292-298. doi:10.1001/jamasurg. 2016.4952

33. Memtsoudis SG, Cozowicz C, Bekeris J, et al. Anaesthetic care of patients undergoing primary hip and knee arthroplasty: consensus recommendations from the International Consensus on Anaesthesia-Related Outcomes after Surgery group (ICAROS) based on a systematic review and meta-analysis. $\mathrm{Br} J$ Anaesth. 2019;123(3):269-287. doi:10.1016/j.bja.2019.05.042

34. Ilfeld BM, Le LT, Meyer RS, et al. Ambulatory continuous femoral nerve blocks decrease time to discharge readiness after tricompartment total knee arthroplasty: a randomized, triple-masked, placebo-controlled study. Anesthesiology. 2008;108(4):703-713. doi:10.1097/ALN.0b013e318167af46

35. Safa B, Gollish J, Haslam L, McCartney CJ. Comparing the effects of single shot sciatic nerve block versus posterior capsule local anesthetic infiltration on analgesia and functional outcome after total knee arthroplasty: a prospective, randomized, double-blinded, controlled trial. J Arthroplasty. 2014;29(6):1149-1153. doi:10.1016/j.arth.2013. 11.020

36. Abdallah FW, Chan VW, Gandhi R, Koshkin A, Abbas S, Brull R. The analgesic effects of proximal, distal, or no sciatic nerve block on posterior knee pain after total knee arthroplasty: a double-blind placebo-controlled randomized trial. Anesthesiology. 2014;121 (6):1302-1310. doi:10.1097/ALN.0000000000000406

37. Pham Dang C, Gautheron E, Guilley J, et al. The value of adding sciatic block to continuous femoral block for analgesia after total knee replacement. Reg Anesth Pain Med. 2005;30(2):128-133. doi:10.1016/j.rapm.2004.11.009

38. Morin AM, Kratz CD, Eberhart LH, et al. Postoperative analgesia and functional recovery after total-knee replacement: comparison of a continuous posterior lumbar plexus (psoas compartment) block, a continuous femoral nerve block, and the combination of a continuous femoral and sciatic nerve block. Reg Anesth Pain Med. 2005;30(5):434-445. doi:10.1016/j.rapm.2005.05.006

39. Wegener JT, van Ooij B, van Dijk CN, Hollmann MW, Preckel B, Stevens MF. Value of single-injection or continuous sciatic nerve block in addition to a continuous femoral nerve block in patients undergoing total knee arthroplasty: a prospective, randomized, controlled trial. Reg Anesth Pain Med. 2011;36(5):481-488. doi:10.1097/ AAP.0b013e318228c33a

40. Eccles CJ, Swiergosz AM, Smith AF, Bhimani SJ, Smith LS, Malkani AL. Decreased opioid consumption and length of stay using an IPACK and adductor canal nerve block following total knee arthroplasty. J Knee Surg. 2019. doi:10.1055/s-0039-1700840

41. Runge C, Moriggl B, Børglum J, Bendtsen TF. The spread of ultrasound-guided injectate from the adductor canal to the genicular branch of the posterior obturator nerve and the popliteal plexus: a Cadaveric Study. Reg Anesth Pain Med. 2017;42(6):725-730. doi:10.1097/AAP.0000000000000675

42. Raddaoui K, Radhouani M, Bargaoui A, et al. Adductor canal block: effect of volume of injectate on sciatic extension. Saudi J Anaesth. 2020;14(1):33-37. doi:10.4103/sja.SJA_410_19

43. Sun C, Zhang X, Song F, et al. Is continuous catheter adductor canal block better than single-shot canal adductor canal block in primary total knee arthroplasty?: a GRADE analysis of the evidence through a systematic review and meta-analysis. Medicine (Baltimore). 2020;99(20):e20320. doi:10.1097/MD.0000000000020320

44. Niesen AD, Harris DJ, Johnson CS, et al. Interspace between Popliteal Artery and posterior Capsule of the Knee (IPACK) Injectate Spread: a Cadaver Study. J Ultrasound Med. 2019;38 (3):741-745. doi:10.1002/jum.14761

45. Runge C, Bjørn S, Jensen JM, et al. The analgesic effect of a popliteal plexus blockade after total knee arthroplasty: a feasibility study. Acta Anaesthesiol Scand. 2018;62(8):1127-1132. doi:10.1111/aas.13145 
46. Sankineani SR, Reddy ARC, Eachempati KK, Jangale A, Gurava reddy AV. Comparison of adductor canal block and IPACK block (interspace between the popliteal artery and the capsule of the posterior knee) with adductor canal block alone after total knee arthroplasty: a prospective control trial on pain and knee function in immediate postoperative period. Eur J Orthop Surg Traumatol. 2018;28(7):1391-1395. doi:10.1007/s00590-018-2218-7

47. Kampitak W, Tansatit T, Tanavalee A, Ngarmukos S. Optimal location of local anesthetic injection in the interspace between the popliteal artery and posterior capsule of the knee (iPACK) for posterior knee pain after total knee arthroplasty: an anatomical and clinical study. Korean J Anesthesiol. 2019;72(5):486-494. doi:10.4097/ kja. 19060

48. El-Boghdadly K, Pawa A, Chin KJ. Local anesthetic systemic toxicity: current perspectives. Local Reg Anesth. 2018;11:35-44. doi:10.2147/LRA.S154512
49. $\mathrm{Cb}$ B, Gr S. Local anesthetics. In: Miller's Anesthesia.. eighth ed. Philadelphia: Elsevier; 2015:1043.

50. Stoto MA. Drug safety meta-analysis: promises and pitfalls. Drug Saf. 2015;38(3):233-243. doi:10.1007/s40264-015-0268-x

51. Nguyen KT, Marcelino R, Jagannathan N, Suresh S, Sawardekar A. Infiltration between popliteal artery and capsule of the knee block to augment continuous femoral nerve catheter for adolescent anterior cruciate ligament reconstruction: a case series. A a Pract. 2020;14 (2):37-39. doi:10.1213/XAA.0000000000001135

\section{Publish your work in this journal}

Local and Regional Anesthesia is an international, peer-reviewed, open access journal publishing on the development, pharmacology, delivery and targeting and clinical use of local and regional anesthetics and analgesics. The journal welcomes submitted papers covering original research, basic science, clinical studies, reviews \& evaluations, guidelines, expert opinion and commentary, case reports and extended reports. The manuscript management system is completely online and includes a very quick and fair peer-review system, which is all easy to use. Visit http://www.dovepress.com/testimonials. php to read real quotes from published authors. 